\title{
Veterans stepping forward for treatment of operational stress injuries
}

Published at www.cmaj.ca on Mar. 16

$\mathrm{H}$ owie McGregor joined the army when he was 18 years old. He shipped out as a paratrooper before his 19th birthday and saw active duty for nearly 30 years.

When he wasn't in combat, McGregor trained other paratroopers how to jump from the bellies of bombers. Then he became a pilot, flying "gliders as big as planes."

McGregor, 85, says it has taken decades to admit that he may have lingering problems from his service days.

"I think I've got post traumatic stress disorder in spades," he told a public seminar - Invisible Wounds: Recovery for Veterans and their Families on operational stress injuries at the Royal Ottawa Mental Health Care Centre in Ottawa, Ontario, on Mar. 11. "And I need a lot of help," added an emotionally distraught McGregor.

Dr. Michele Boivin, one of three psychologists who are part of a 10-member Royal Ottawa clinic for operational stress injuries, was encouraged by McGregor's admission.

"We've actually had several veterans in the elderly age range who are now coming forward and I think that's a combination of a lot of things," Boivin says. "Certainly with the current conflicts in the news, that is making their experiences relevant for people again. Also our knowledge has really increased since the 1980s when PTSD [post-traumatic stress disorder] became an official diagnosis ... It's really just in the past couple of decades that we've begun to understand more about what
PTSD really is and how to go about treating it."

The Royal Ottawa clinic is 1 of 10 across Canada funded by the Department of Veterans Affairs to offer help to both current and retired military personnel and

attitudes within both the military and general populations.

Patrick Sudrau waited two years before seeking help after serving as a medic in Croatia in 1993.

"In 1995, I realized I still had some issues," he says. He still can't talk about the eight traumatic events "that I can remember" experiencing while serving with the Canadian Armed Forces.

Sudrau is now a full-time peer support coordinator with the Operational Stress Injuries Support Service jointly run by Veterans Affairs Canada and the Department of National Defence. It is a peer support program for soldiers, veterans and their families that is complementary to the Royal Ottawa clinic.

Sudrau has found assisting other soldiers to be therapeutic. "I was able to develop more resiliency and come to peace with my issues and give back what I've learned to support and help other servicemen."

But many others struggle to cope with operational stress

Royal Canadian Mounted Police officers who have, or suspect they have, an operational stress injury.

Boivin says post-traumatic stress disorder is more common than other operational stress injury such as anxiety disorder, depression or addiction.

"The risk of developing PTSD is about three times higher for people in the military or law enforcement than for the general population," she says. "So we know a lot about who is at risk for PTSD, but there is still a lot we don't know."

Boivin adds that it is likely posttraumatic stress disorder is underreported as a consequence of barriers and injuries on their own or refuse to admit they need help, he adds, noting that PTSD sufferers are six times more likely to have thoughts of suicide.

"There's probably more people out there who are not coming forward, but they eventually will through culture change, and education. [That] eventually will lead to better results," Sudrau says. "Everybody has their own threshold. Everybody is different. Some people wait longer and you have to remember, back in Howie's day, the culture was different. So for him to come forward now, he's really a strong man."

Since opening in February 2009, the Ottawa operational stress injuries clinic 
has assessed and referred about 140 clients. "We hope that, as that word gets out, that people will know they are not alone," Boivin says. "That is always one of the difficult barriers in seeking services. If someone feels they are the only person with this problem they are less likely to seek help. We hope that people become aware that this is a more common problem than they might think. That might reduce some of those barriers and they'll have improved access to care." — Becky Rynor, Ottawa, Ont.

DOI:10.1503/cmaj.109-3213
Editor's note: Second of a series on mental health and the military.

Part 1: Where "stigma leaves the room." (CMAJ 2010. DOI:10.1503 /cmaj.109-3197) 